\section{Anemia ferropriva em escolares da primeira série do ensino fundamental da rede pública de educação de uma região de Brasília, DF}

\section{Iron deficiency anemia in first grade students from public schools in a region of Brasilia, DF}

\section{Gracy Santos Heijblom \\ Leonor Maria Pacheco Santos}

Departamento de Nutrição, Faculdade de Ciências da Saúde, Universidade de Brasília

Financiamento: Ministério da Saúde (convênio com a UnB)

Contribuição dos autores: LMPS e GSH atuaram como responsáveis pela concepção do projeto, elaboração dos instrumentos, coleta, análise e interpretação dos dados; são igualmente responsáveis pela autoria e revisão final do texto. As autoras declaram que não há conflito de interesses. Correspondência: Leonor M. P. Santos. 'Departamento de Nutrição, Faculdade de Ciências da Saúde, Universidade de Brasília. Campus Universitário Darcy Ribeiro. Asa Norte, Brasília, DF, CEP: 70910-900.E-mail: leopac@unb.br

\section{Resumo}

A anemia ferropriva é a deficiência de micronutriente mais prevalente no mundo. É particularmente deletéria em escolares, pois crianças anêmicas são sonolentas e prestam menos atenção. Como conseqüência, pode levar ao alto absenteísmo e baixo rendimento escolar. Realizou-se um estudo transversal com amostra probabilística de 424 alunos de 6 a 11 anos, da $1^{\text {a }}$ série do ensino fundamental de escolas públicas da Regional Norte de Saúde de Brasília. Os objetivos foram avaliar a prevalência de anemia e comparar o resultado obtido em 2004 com aquele de estudo similar realizado na mesma área em 1998. A hemoglobina (Hb) foi determinada em amostra de sangue digital, usando Hemocue $^{\circledR}$. Dois pontos de corte foram adotados para classificar anemia, ambos estabelecidos pela Organização Mundial da Saúde: $\mathrm{Hb}<11,5 \mathrm{~g} / \mathrm{dL}$ e $\mathrm{Hb}<12,0$ g/dL. O retardo pondero-estatural foi diagnosticado quando os indicadores Altura/Idade (A/ I), Peso/Idade (P/I) e Peso/Altura (P/A) encontravam-se abaixo de -2,0 desviospadrão da referência NCHS. A prevalência de anemia foi de $12,5 \%$ e $11,9 \%(\mathrm{Hb}<11,5 \mathrm{~g} /$ $\mathrm{dL})$ e de $26,9 \%$ e $21,5 \%(\mathrm{Hb}<12,0 \mathrm{~g} / \mathrm{dL})$ em 2004 e 1998, respectivamente. A prevalência de anemia aumentou entre os inquéritos, mas não houve diferença estatisticamente significativa. Tampouco houve associação estatisticamente significativa entre retardo do crescimento e anemia. Observa-se presença importante de anemia entre os escolares, o que justifica a implementação de políticas públicas específicas para o enfrentamento do problema, a exemplo da fortificação de farinhas de trigo e milho com ferro, tornada obrigatória em 2004.

Palavras-chave: Anemia. Crianças. Saúde Escolar. Estudos Transversais. 


\section{Abstract}

Iron deficiency anemia (IDA) is the most prevalent micronutrient deficiency in the world. In school age children, IDA is particularly deleterious: anemic children are sleepy and pay less attention. As a consequence, anemia can lead to high absenteeism and lower school performance. A cross-sectional survey was conducted in a representative sample of 424 randomly selected first graders (ages 6 to 11 years) from public schools located in the Northern Public Health Region of Brasília. The study objectives were to: (a) determine the prevalence of anemia; (b) compare the results obtained in 2004 to those of a similar survey conducted in the same area in 1998. Hemoglobin $(\mathrm{Hb})$ was determined in finger-prick blood samples using Hemocue ${ }^{\oplus}$. Two cut-off points were used to classify anemia, both established by the WHO: $\mathrm{Hb}<11.5 \mathrm{~g} / \mathrm{dL}$ and $\mathrm{Hb}<12.0 \mathrm{~g} / \mathrm{dL}$. The height/age, weight/age and weight/height indicators below -2.0 standard deviations from the NCHS reference were used to indicate growth retardation. Prevalence of anemia was $12.5 \%$ and $11.9 \%(\mathrm{Hb}<11.5 \mathrm{~g} /$ $\mathrm{dL})$ and $26.9 \%$ and $21.5 \%(\mathrm{Hb}<12.0 \mathrm{~g} / \mathrm{dL})$ in 2004 and 1998 respectively. The prevalence of anemia increased between the surveys, but the difference was not significant. There was no statistically significant association between growth retardation and anemia. A high prevalence of anemia was observed, which justifies the implementation of public policies to fight the problem, such as the fortification of wheat and corn flours with iron, mandatory since 2004 .

Keywords: Anemia. Children. School Health. Cross-Sectional Studies.

\section{Introdução}

O ferro é um dos mais abundantes elementos na crosta terrestre, mas apesar disto representa a mais comum e mais grave deficiência de micronutrientes em todo o mundo $^{1}$. Estima-se que há mais de dois bilhões de pessoas no mundo com anemia, o que representa $1 / 3$ da população mundial. Este agravo não é problema exclusivo de populações carentes, sendo considerado uma endemia em expansão em todos os segmentos sociais. A prevalência da anemia é maior em países em desenvolvimento. Os grupos de maior risco para desenvolver anemia são mulheres grávidas e crianças, particularmente as menores de dois anos de idade ${ }^{2}$. Diferentemente do observado com relação às deficiências de vitamina $\mathrm{A}$, iodo, proteína e energia, não há relato de progresso significativo no controle dos níveis endêmicos de anemia ferropriva na população infantil em países em desenvolvimento ${ }^{3}$.

A anemia é definida como a condição na qual o conteúdo de hemoglobina no sangue está abaixo do esperado, sendo evidenciada pela redução da qualidade ou quantidade de células vermelhas no sangue, como resultado da carência de um ou mais nutrientes essenciais, seja qual for a causa desta deficiência ${ }^{1,4}$. A anemia ferropriva pode ser considerada o estágio final de um longo período de balanço negativo do ferro. A deficiência de ferro e anemia ferropriva resulta do desequilíbrio no balanço entre a quantidade de ferro biodisponível absorvido na dieta e a necessidade do organismo, ou seja, o suprimento de ferro é insuficiente para a síntese normal de componentes que dependem deste mineral ${ }^{5}$.

Atualmente é reconhecido que não só a anemia, mas também a deficiência leve ou moderada de ferro podem causar efeitos adversos no desenvolvimento cognitivo, na capacidade imunológica e na capacidade de trabalho e produtividade ${ }^{6}$. Durante a gravidez, a deficiência de ferro aumenta o risco de prematuridade, mor- 
talidade materna e de crianças no período pré-natal e perinatal. A deficiência de ferro altera a função tiroidiana e também a produção e o metabolismo das catecolaminas e de outros neurotransmissores, além de aumentar, nos indivíduos anêmicos, a capacidade de absorção de metais pesados tóxicos ${ }^{6}$.

Diversos estudos em diferentes regiões do mundo registram a ocorrência da anemia como problema de saúde pública ${ }^{7}$. O Unicef em 2004 declarou que na maioria dos países em desenvolvimento a deficiência de ferro em crianças é mais a regra do que a exceção. Estudo conduzido pela OPAS, na Guiana e no Caribe, mostrou grande heterogeneidade das prevalências de anemia ferropriva em escolares, variando de 0,8\% nas Bahamas a 57,0\% na Guiana. República Dominicana, Barbados e Ilhas Cayman apresentaram uma prevalência superior a $25,0 \%^{8}$. Na região metropolitana de Caracas, Venezuela, a anemia detectada pelo método padrão da cianometahemoglobina em escolares com idades de 7,11 e 15 anos aumentou de $6 \%$ para $13 \%$ entre 1989 e $1992^{9}$.

No Brasil ainda não existem dados nacionais sobre a prevalência da anemia ferropriva. Foram identificados oito levantamentos com escolares entre $1990 \mathrm{e}$ $2000^{10-13}$, nos quais crianças de 6 a 14 foram classificadas com anemia ferropriva se a concentração de hemoglobina fosse $\mathrm{Hb}<12,0 \mathrm{~g} / \mathrm{dL}$, segundo definição da $\mathrm{OMS}^{14}$ que vigorou até outubro de 1998. Foram encontradas as seguintes prevalências de anemia: São Lourenço da Mata, PE, 43,1\% $(1997)^{10}$; Aracaju, 26,7\% (1998) ${ }^{10}$ e Maceió, $25,4 \%(2000)^{10}$. Em Porto Velho, RO, encontrou-se $30,9 \%$ de anemia $(1990)^{11}$; em Osasco, SP, 51,0\% (1991) ${ }^{11}$; e em Santos, SP, $27,8 \%, 11,3 \%$ e $5,6 \%$ nos escolares da $1^{\text {a }}, 5^{\text {a }}$ e $8^{\text {a }}$ séries, respectivamente $(1996)^{11}$. Em uma região de Brasília foi encontrada prevalência de 21,5\% (1998) ${ }^{12}$, e em Londrina $41,3 \%(1998 / 9)^{13}$.

Considerando-se a existência de poucos levantamentos sobre anemia em escolares na região centro-oeste, este estudo foi pla- nejado e conduzido para estimar a sua prevalência nos alunos da primeira série do ensino fundamental do Distrito Federal.

\section{Material e Métodos}

No ano de 2004, foi realizado um estudo de corte transversal em uma amostra representativa de alunos da primeira série do ensino fundamental das escolas públicas da Regional Norte de Saúde do DF. O trabalho de campo foi desenvolvido no período de 07 de maio a 06 de agosto de 2004. O inquérito foi desenvolvido na mesma região da Pesquisa "Nutrição Escolar Avaliação do estado nutricional de escolares das unidades da Rede Oficial de Ensino, localizadas na Regional Norte do Distrito Federal", realizada em 1998, que avaliou o estado nutricional, os hábitos alimentares e a prevalência de anemia dos escolares $^{12}$. Empregou-se amostragem e metodologia semelhantes, possibilitando verificar tendência temporal na condição nutricional dos escolares.

A população do estudo foi composta pelos alunos da $1^{\text {a }}$ série do ensino fundamental matriculados no ano de 2004 nas escolas urbanas da rede oficial de ensino localizadas na Regional Norte de Saúde do DF - composta pelas localidades do Cruzeiro, Varjão, Asa Norte e Paranoá. Ao todo eram 28 escolas públicas, que totalizavam 3.512 alunos matriculados, segundo dados do Censo Escolar de 2004, fornecidos pela Secretaria de Educação do Distrito Federal (SEDF).

A falta de um cadastro prévio de alunos impossibilitou a amostragem aleatória simples. As informações disponibilizadas pela SEDF foram a identificação da escola, o número de turmas por escola que, na primeira série, variava de uma a cinco turmas - e o total de alunos. Optouse por uma amostragem por conglomerado, em dois estágios: no primeiro estágio, foram sorteadas aleatoriamente as escolas, e no segundo foram sorteadas duas turmas para cada escola; todos os alunos da turma selecionada foram pesquisados. $\mathrm{O}$ to- 
tal de escolas foi distribuído por localidade de forma proporcional ao número de escolas por localidade. Ao todo foram selecionadas 22 turmas de 13 escolas, sendo 7 escolas na Asa Norte, 3 no Paranoá, 2 no Cruzeiro e 1 no Varjão. Não existindo grande heterogeneidade no número de turmas por escola, não houve necessidade de calcular fatores de expansão da amostra.

Calculou-se o tamanho da amostra supondo prevalência de anemia de 21,5\% (valor encontrado em 1998) ${ }^{14}$, erro aceitável de $\pm 5 \%$, 95\% de grau de confiança, amostra aleatória simples e população finita (3.512 alunos), resultando em 242 crianças. Foi necessário empregar o Deff (design effect) para corrigir o tamanho da amostra levando em conta o efeito do desenho amostral utilizado - conglomerado em dois estágios - em relação a uma amostra aleatória simples do mesmo tamanho. O Deff calculado foi de 1,754 ajustando o tamanho da amostra desejada para 425 crianças. Foi planejada uma reserva de alunos supondo possíveis perdas no processo de coleta dos dados. O baixo valor obtido no cálculo do Deff indicou não haver comprometimento da estimativa de erro.

De um universo de 3.512 alunos de 28 escolas existentes nesta região, foram sorteados 605. Porém foram autorizados pelos pais 441 alunos e efetivamente avaliados 424 alunos. Os 17 alunos autorizados e que não foram avaliados não estavam presentes no dia marcado para a avaliação.

Foram aplicados questionários com perguntas sobre bens básicos, dados socioeconômicos e freqüência alimentar feitas individualmente, pela equipe da pesquisa, antes ou depois do processo da avaliação antropométrica. Da mesma forma que na pesquisa de 1998, o questionário de freqüência de consumo alimentar, educação dos pais e de bens básicos também foi enviado aos pais, para verificar a fidedignidade das informações repassadas pelas crianças $^{12}$.

Foi empregada a classificação socioeconômica da ABIPEME, que consiste na definição de alguns indicadores escolhidos com base na sua correlação com a renda familiar, para os quais é definida uma escala de pontos, que traduz o status socioeconômico da família em classes sociais que variam de $\mathrm{A}$ a $\mathrm{E}$, a saber: Classe A - Alta (89 pontos ou mais); Classe B - Média alta (de 59 a 88 pontos); Classe $\mathrm{C}$ - Média média (de 35 a 58 pontos); Classe D - Média pobre (de 20 a 24 pontos) e Classe E - Pobre. ${ }^{15} \mathrm{~A}$ análise da escolaridade dos pais considerou os adultos com menos de 3 anos de estudo na categoria "Não estudou/ primário incompleto"; as demais categorias são auto-explicativas.

O diagnóstico da anemia foi realizado por meio da dosagem da concentração sangüínea de hemoglobina pelo método da cianometahemoglobina, utilizando-se fotômetro de alta precisão HemoCue ${ }^{\circledR}$. O sangue foi colhido por punção digital com lanceta descartável e introduzido no aparelho, utilizando-se lâmina apropriada, também descartável, e em poucos segundos foi feita a leitura do valor da hemoglobina apresentado digitalmente ${ }^{16}$.

A anemia ferropriva foi definida mediante dois pontos de corte para a hemoglobina, ambos propostos pela OMS. A recomendação mais recente indica anemia quando a concentração de hemoglobina é de $\mathrm{Hb}<11,5 \mathrm{~g} / \mathrm{dL}$ para crianças na faixa etária de 6 a 11 anos, e mantém $\mathrm{Hb}<12,0 \mathrm{~g} /$ dL entre os 12 e 14 anos $^{6}$. A indicação anterior, de $\mathrm{Hb}<12,0 \mathrm{~g} / \mathrm{dL}$ para escolares de 6 a 14 anos, vigorou até outubro de $1998^{14}$. As duas classificações foram usadas para comparar este estudo com os dados coletados em 1998 na mesma população e com outros estudos publicados em data anterior à mudança. No entanto, só foram encaminhadas para tratamento nos serviços de saúde as crianças diagnosticadas com hemoglobina $<11,5 \mathrm{~g} / \mathrm{dL}$.

Peso e altura foram coletados conforme técnicas sugeridas pelo Ministério da Saúde (SISVAN). O peso foi obtido em kilograma, utilizando-se balança digital, portátil SOEHNLE ${ }^{\circledR}$, modelo BE-04, com capacidade de $150 \mathrm{~kg}$ e precisão de $100 \mathrm{~g}$. A 
estatura foi verificada com estadiômetro afixado à parede (SECA Modelo 200) com precisão de $0,1 \mathrm{~cm}$. Os dados antropométricos foram analisados com o módulo Epinut do EpiInfo 2002 ${ }^{17}$, utilizando os indicadores peso/idade $(\mathrm{P} / \mathrm{I})$, altura/idade (A/I) e peso/altura (P/A). Considerou-se o ponto de corte -2,0 desvios-padrão da referência do National Center for Health Statistics (NCHS) como indicativo de déficit e + 2,0 desvios padrão de P/A para excesso de peso $^{18}$. A análise estatística foi realizada utilizando-se também o programa EPI-Info 2002 for Windows.

$\mathrm{Na}$ associação entre variáveis utilizouse o teste do Qui-quadrado de Pearson, adotando-se nível de significância de 5\% ou o teste de probabilidade exata de Fisher quando apropriado. Para as prevalências foram calculadas estimativas pontuais e intervalares, correspondendo ao intervalo de confiança de $95 \%$.

O Protocolo de pesquisa foi aprovado pelo Comitê de Ética em Pesquisa da Faculdade de Saúde da Universidade de Brasília. A pesquisa só foi realizada pelos alunos autorizados pelos pais ou responsáveis após esclarecimentos e assinatura do "Termo de consentimento livre e esclarecido".

\section{Resultados e Discussão}

Foram avaliados 424 alunos, sendo 212 do sexo masculino e 212 do sexo feminino. A idade variou de cinco anos e dez meses a onze anos e oito meses, com média de 8 anos. As principais características socioeconômicas das famílias da pesquisa estão incluídas na Tabela 1. Aproximadamente $40 \%$ dos pais e $35 \%$ das mães não estudaram ou não completaram a $4^{\mathrm{a}}$ série do ensino fundamental. Em torno de $42 \%$ das famílias pertencem à classe $\mathrm{D}$ (Média pobre).

Nas 424 crianças a prevalência de anemia de acordo com o novo ponto de corte

Tabela 1 - Características sociais e econômicas de escolares da primeira série do ensino fundamental da rede pública da regional Norte de Saúde do DF, Brasil, 2004.

Table 1 - Socio-economic features of first-graders from public schools in the North Health Region, DF, Brazil, 2004.

\begin{tabular}{lc} 
Variáveis sociais e econômicas & Freqüência (\%) \\
\hline Nível de escolaridade do pai ( $\mathrm{n}=413)$ & \\
Não estudou/ primário incompleto & 39,7 \\
Primário completo/ Ginasial incompleto & 25,2 \\
Ginasial completo/ Colegial incompleto & 16,2 \\
Colegial completo/ Universitário incompleto & 13,1 \\
Universitário completo & 5,8 \\
Total & 100,0 \\
Nível de escolaridade da mãe ( $\mathrm{n}=412)$ & \\
Não estudou/ primário incompleto & 35,2 \\
Primário completo/ Ginasial incompleto & 29,1 \\
Ginasial completo/ Colegial incompleto & 17,2 \\
Colegial completo/ Universitário incompleto & 16,0 \\
Universitário completo & 2,4 \\
Total & 100,0 \\
Classe social das famílias ( $\mathrm{n}=391$ ) & \\
Classe A (Alta) & 0,3 \\
Classe B (Média alta) & 10,2 \\
Classe C (Média média) & 34,0 \\
Classe D (Média pobre) & 41,7 \\
Classe E (Pobre) & 13,8 \\
Total & 100,0 \\
\hline
\end{tabular}


$(\mathrm{Hb}<11,5 \mathrm{~g} / \mathrm{dL})$ foi de 12,5\% $\left(\mathrm{IC}_{95 \%} 9,6\right.$ $16,1)$, porém, conforme o ponto de corte anterior $(\mathrm{Hb}<12,0 \mathrm{~g} / \mathrm{dL})$ a prevalência de anemia seria de $26,9 \%\left(\mathrm{IC}_{95 \%} 22,8-31,4 \%\right.$ ) (Tabela 2). O valor mínimo de hemoglobina encontrado foi 8,5 g/dL e o máximo $16,0 \mathrm{~g} / \mathrm{dL}$.

No sexo masculino e feminino, as prevalências foram semelhantes (Tabela 2) e os valores médios da hemoglobina observados foram iguais: 12,6 g/dL (desvio padrão $1,11 \mathrm{~g} / \mathrm{dL}$ e $1,13 \mathrm{~g} / \mathrm{dL}$ respectivamente - Teste $\mathrm{T}=0,12 \mathrm{p}$ valor $=0,90)$. Os resultados dos valores de prevalência de anemia e valores médios de hemoglobina nos dois sexos indicam que na faixa etária pesquisada o sexo não é uma variável importante na etiologia da anemia ferropriva.

Os resultados de alguns dos estudos epidemiológicos realizados em escolares no Brasil empregando o novo critério $(\mathrm{Hb}<11,5 \mathrm{~g} / \mathrm{dL})$ se assemelham aos $12,5 \%$ observados neste estudo: $13,0 \%$ no Rio de Janeiro (1999) ${ }^{19}$ e 9,9\% em Maceió (2000) ${ }^{20}$. Já outros dois estudos relataram prevalências mais elevadas: em escolares de 6 a 12 anos de famílias de baixa renda numa área rural do Paraná (1998/9) a prevalência foi de $25,7 \%^{21}$, e na área urbana de Belo Horizonte, em 2002, encontrou-se em escolares do ensino público $30,4 \%$ de anemia na faixa etária de 6 a 7 anos, e 25,1\% na faixa etária de 8 a 11 anos. ${ }^{22}$ De modo geral, no Brasil as carências nutricionais apresentam-se mais freqüentes nas áreas rurais do que nas urbanas. Neste sentido, o resultado de Belo Horizonte se destaca com prevalência maior que na zona rural, superando em quase três vezes aquela encontrado em Maceió.
De acordo com critérios propostos pela OMS, o esperado ou aceitável seria uma prevalência de até $5 \%$ em todos os grupos etários para ambos os sexos. Para mensurar a magnitude da anemia ferropriva como um problema de saúde pública, a OMS considera o problema como "Leve" se a prevalência se situa na faixa de 5,0 a 19,9\%, "Moderado" de 20,0 a 39,9\%, e “Grave" se maior ou igual a $40 \%^{6}$. Portanto, os resultados encontrados no DF, RJ e Maceió podem ser classificados na primeira categoria ("Leve"), enquanto os do Paraná e Belo Horizonte na segunda ("Moderado").

Este cenário aponta para a necessidade de intervenção em ações que envolvam as escolas, os escolares, os familiares e os serviços de saúde, para reduzir a prevalência de anemia apontada em pesquisas em diferentes regiões brasileiras.

Neste sentido, a educação e orientação nutricional têm papel de destaque, como uma ferramenta potente para que a população, orientada, possa utilizar de maneira mais adequada os alimentos. Contudo, uma pesquisa realizada por Boog $(1999)^{23}$ em Unidades Básicas de Saúde mostra que médicos e enfermeiros têm dificuldade em identificar problemas alimentares nos pacientes; e consideram o acesso à alimentação adequada uma questão que foge aos objetivos do serviço de saúde, tornando ineficiente ou mesmo inexistente, nos referidos serviços, orientações sobre alimentação e nutrição.

A prevalência de anemia, apesar de mais elevada, não diferiu significativamente entre as crianças eutróficas e as que apresentaram retardo pondo-estatural (abaixo de -2DP), em relação aos indica-

Tabela 2 - Prevalência da anemia segundo diferentes pontos de corte de hemoglobina $(\mathrm{Hb})$ em escolares da primeira série do ensino fundamental da rede pública da Regional Norte de Saúde do DF, Brasil, 2004.

Table 2 - Prevalence of anemia according to different hemoglobin $(\mathrm{Hb})$ cut-off points among first-graders from public schools in the North Health Region, DF, Brazil, 2004.

\begin{tabular}{lccc}
\hline Critério diagnóstico & Total $\left[\mathrm{IC}_{95 \%}\right](\mathrm{n}=424)$ & Meninos $(\mathrm{n}=212)$ & Meninas $(\mathrm{n}=212)$ \\
\hline $\mathrm{Hb}<11,5 \mathrm{~g} / \mathrm{dL}$ & $12,5 \%[9,6-16,1]$ & $13,7 \%$ & $11,3 \%$ \\
$\mathrm{Hb}<12,0 \mathrm{~g} / \mathrm{dL}$ & $26,9 \%[22,8-31,4]$ & $27,8 \%$ & $25,9 \%$ \\
\hline
\end{tabular}


dores altura para idade (A/I), peso para idade $(\mathrm{P} / \mathrm{I})$ e peso para altura (P/A) (Tabela 3$)$. A relação entre anemia e sobrepeso também não mostrou associação. Resultados semelhantes, no que se refere à anemia $\mathrm{e}$ a retardo do crescimento, foram encontrados em escolares de Maceió ${ }^{20}$.

Em 1998, estudo conduzido em 302 escolares na mesma região de Brasília ${ }^{12}$ mostrou uma prevalência menor de anemia, independente do critério de classificação empregado (Tabela 4). Considerando-se o critério tradicional, observa-se bem a tendência de crescimento da anemia, com aumento de 5,4 pontos percentuais, o que, contudo, não representou uma diferença estatisticamente significativa ( $p$ valor $=0,11$ ). Tampouco a comparação entre as médias $\mathrm{de} \mathrm{Hb}$ entre os dois estudos revelou um resultado significativo $(t=1,311 \mathrm{p}$ valor $=0,19)$. Estudos empregando amostras maiores talvez pudessem evidenciar alguma significância.
Alguns autores têm relatado a tendência crescente da anemia em vários países e no Brasil ${ }^{9,24,25}$ ainda que esforços tenham sido empregados em programas de redução desta carência de micronutrientes. Yip $^{26}$ considera que a limitação em atingir as metas de redução de anemia em diversos grupos populacionais, em comparação com o relativo sucesso no controle da deficiência de outros micronutrientes, como vitamina A e iodo, está relacionada a falhas na implementação e na efetividade das intervenções, além da ineficiência da comunicação. Nantel e Tontisirin ${ }^{27}$ argumentam que nos últimos 30 anos as questões operacionais dos programas de combate à deficiência de ferro têm sido negligenciadas, o que explica o modesto avanço nesta área.

A fortificação de alimentos é uma medida que tem obtido sucesso na redução da prevalência de anemia em estudos conduzidos no Brasil e no exterior ${ }^{28,29}$. Esta é

Tabela 3 - Prevalência de anemia segundo os indicadores antropométricos em escolares da primeira série do ensino fundamental da rede pública da Regional Norte de Saúde do DF, Brasil, 2004.

Table 3 - Prevalence of anemia according to anthropometric indicators among first-graders from public schools in the North Health Region, DF, Brazil, 2004.

\begin{tabular}{lccc}
\hline & \multicolumn{3}{c}{$\begin{array}{c}\text { Prevalência de anemia (\%) } \\
\text { (nível de hemoglobina }<11,5 \mathrm{~g} / \mathrm{dL})\end{array}$} \\
\hline Classificação antropométrica & $\mathrm{A} / \mathrm{I}(\mathrm{n}=424)$ & $\mathrm{P} / \mathrm{l}(\mathrm{n}=394)$ & $\mathrm{P} / \mathrm{A}(\mathrm{n}=392)$ \\
Deficiente $(<-2 \mathrm{DP})$ & $25,0^{\mathrm{a}}$ & $28,6^{\mathrm{b}}$ & $25,0^{\mathrm{c}}$ \\
Eutrófico $(\geq-2 \mathrm{DP})$ & $12,0^{\mathrm{a}}$ & $12,1^{\mathrm{b}}$ & $12,6^{\mathrm{c}}$ \\
\hline Teste Fisher & ${ }^{\mathrm{a}} \mathrm{p}=0,126$ & ${ }^{\mathrm{b}} \mathrm{p}=0,087$ & ${ }^{\mathrm{c}} \mathrm{p}=0,421$ \\
\hline
\end{tabular}

Tabela 4 - Comparação da prevalência de anemia e da média de hemoglobina em escolares da primeira série do ensino fundamental da Regional Norte de Saúde do DF, Brasil, 1998 - 2004. Table 4 - Comparison of the prevalence of anemia and mean hemoglobin among first-graders from public schools in the North Health Region, DF, Brasil, 1998 - 2004.

\begin{tabular}{lcccc}
\hline Prevalência de anemia & $1998^{\mathrm{d}}$ & 2004 & Qui $^{2}$ & Valor \\
\hline $\mathrm{Hb}<12,0 \mathrm{~g} / \mathrm{dL}$ & $21,5 \%$ & $26,9 \%$ & 2,45 & 0,11 \\
$\mathrm{Hb}<11,5 \mathrm{~g} / \mathrm{dL}$ & $11,9 \%$ & $12,5 \%$ & 0,01 & 0,91 \\
Valores médios & $1998^{\mathrm{d}}$ & 2004 & $t$ & Valor $p$ \\
Média Hb g/dL (desvio padrão) & 12,77 & 12,66 & 1,31 & 0,19 \\
& $(\mathrm{DP}=1,18)$ & $(\mathrm{DP}=1,12)$ & & \\
\hline
\end{tabular}

${ }^{\mathrm{d}}$ Fonte: referência 12 
uma forma fácil, segura, de baixo custo e efetiva em curto e médio prazo, além de melhorar a qualidade dos alimentos disponibilizados à população ${ }^{30}$. No Brasil, desde junho de 2004 a fortificação das farinhas de trigo e milho com ferro e ácido fólico é obrigatória $^{31}$. Porém é importante que se implementem, conjuntamente, sistemas de monitoramento e fiscalização dos produtos fortificados, além de incentivo às pesquisas de avaliação de efetividade da ação.

\section{Conclusão}

Para ter êxito, a educação não tem que somente estar atenta à infra-estrutura e ao currículo, mas também à educabilidade das crianças. Os esforços empenhados na melhoria da educação passam necessariamente pela freqüência do aluno e pelo aprendizado efetivo; para que isto ocorra, os escolares têm de estar motivados, bem nutridos e alimentados.

Observou-se presença importante de anemia nos escolares ingressantes da rede pública de ensino do DF e pode-se concluir que medidas de monitoramento, preven- ção e controle precisam ser adotadas. A orientação e educação nutricionais, aliadas ao tratamento medicamentoso, devem ser dirigidas a este grupo específico para que os efeitos deletérios da anemia possam ser revertidos. A fortificação das farinhas de trigo e milho com ferro e ácido fólico, recentemente tornada obrigatória no Brasil, pode contribuir para prevenir a anemia ferropriva neste grupo.

\section{Agradecimentos}

À ENSP/Fiocruz e UNB pela cessão de equipamentos e de cubetas para dosagem de $\mathrm{Hb}$. Aos alunos de nutrição da UnB que colaboraram na coleta de dados: Lígia S. Cunha, Mariana Martins, Luciane Morgental, Francilene de Araújo, Melina P. Noronha, Carolina R. Gama, Ana C. B. Guedes Heloísa H. Silva, Aline A. Sousa, Cecília Kinoshita, Élida A. Pereira, Flávio Terra, Maria A. Bacchi, Mariane V. Vieira, Clara F.C. Pinto, Naíra D. Sé, Katiúza Martins, Keila K. Paixão, Laura A. Silva, Rafaela M. Dias, Marcos N. Mata Júnior, Mariana C. Pinheiro, Amanda P. Lima.

\section{Referências}

1. OMS/OPAS. Iron fortification: Guidelines and Recommendations for Latin America and the Caribbean.Washington: OMS/OPAS; 2001.

2. USAID/UNICEF/MI/OPAS/OMS/World Bank. Anemia Prevention and Control: What Works - Part I Program Guidance. USAID, UNICEF, MI, OPAS, OMS, The World Bank. Washington: WHO; 2003.

3. ACC/SCN. Second report on the world nutrition situation. Vol. 1. Global and regional results. ACC/SCN: Geneva; 1992.

4. UNICEF/UNU/WHO/MI Technology Workshop. Preventing Iron Deficiency in Women and Children: technical consensus on key issues and resources for programme advocacy, planning and implementation. UNICEF, New York, 7-9 October; 1998.

5. Cook JD. Determinants of non-heme iron absorption in man. Food technology 1983; 198: 124-6.
6. WHO, UNU, UNICEF. Iron Deficiency Anaemia Assessment, Prevention, and Control: a guide for programme managers. WHO, UNU, UNICEF: Geneva; 2001. Disponível em http://www.who.int/reproductivehealth/docs/anaemia.pdf Acessado em 19 de janeiro de 2007.

7. UNICEF/MI. Vitamin \& Mineral Deficiency: A global progress report. Ottawa/New York: UNICEF/MI; 2004. Disponível em http://www.micronutrient.org/reports/ reports/Full_e.pdf Acessado em 19 de janeiro de 2007.

8. OPAS. Micronutrient deficiencies in the English-speaking Caribbean and Guyana. Pan Amer J Public Health 1998; 4(5): 367-70.

9. Landaeta-Jiménez M, Macias-Tomei C, Fossi M, García MN, Layrisse M, Castellano HM. Tendencia en el crecimiento físico y estado nutricional del niño venezolano. Arch Venez Pueric Pediatr 2002; 65(1); 13-20. Disponível em www.dynabizvenezuela.com/images/ dynabiz/ ID3749/siteinfo/revista12.pdf. Acessado em 19 de janeiro de 2007. 
10. Santos LMP. (org). Bibliografia sobre micronutrientes deficiência de micronutrientes no Brasil 1990-2000. Vol. 2a-Anemia. Brasília: Organização Pan Americana da Saúde; 2002. Disponível: http://www.opas.org.br/ publicacl.cfm. Acessado em 19 de janeiro de 2007.

11. Santos LMP (org). Bibliografia sobre micronutrientes deficiência de micronutrientes no Brasil 1990-2000. Vol. $2 b$-Anemia. Brasília: Organização Pan Americana da Saúde; 2002. Disponível em http://www.opas.org.br/ publicacl.cfm. Acessado em 19 de janeiro de 2007.

12. Ito MK, Schmitz BAS, Furumoto RV, Aquino KK, Miazaki E. Nutrição Escolar: avaliação do estado nutricional de escolares das unidades de ensino localizadas na regional norte de saúde do Distrito Federal. Relatório final. Brasília: UNB; 1998 (mimeo).

13. Miglioranza LHS, Matsuo T, Caballero-Cordoba GM, Dichi JB, Cyrino ES, Oliveira IBN, Martins MS, Polezer N, Dichi I. Anemia prevalence in children and adolescents from educational centers in the outskirts of Londrina, PR, Brazil. Rev Nutr 2002; 15(2): 149-153. Disponível em http://www.scielo.br/pdf/rn/v15n2/11831.pdf. Acessado em 21 de janeiro de 2007.

14. Demayer EM, Adies-Tegman M. The prevalence of anaemia in the world. World Health Statistics Quartely 1985; 38: 302-16.

15. Mattar FN. Análise crítica dos estudos de estratificação socioeconômica de ABA-Abipeme. Revista de Administração 1995; 30(1): 57-74.

16. USAID/PATH. Anemia detection in health services: guidelines for program managers. Seattle, WA: USAID; 1996.

17. CDC/WHO. EPi Info 6, version windows. $A$ World Processing, Database and Statistics Program for Public Health. Atlanta: CDC/Geneva: WHO; 2002.

18. WHO. Measuring Change in nutritional status: guidelines for assessing the nutritional impact of supplementary feeding programs for vulnerable groups. Geneva: WHO; 1983.

19. SMS-RJ (Secretaria Municipal de Saúde do Rio de Janeiro)/SME-RJ (Secretaria Municipal de Educação do Rio de Janeiro). II Pesquisa de Saúde e Nutrição em Escolares. Rio de Janeiro: Prefeitura Municipal do Rio de Janeiro; 2000.
20. Santos CD, Santos LMP, Figueiroa JN, Marroquim PMG, Oliveira MAA. Anemia em escolares da primeira série do ensino fundamental da rede pública de Maceió, Alagoas, Brasil. Cad Saúde Pública 2002; 18(6): 1757-63.

21. Sichieri R, Mathias T, Moura AS. Stunting, high weightfor-height, anemia and dietary intake among Brazilian students from a rural community. Nutrition Res 1996; 16: 201-09.

22. Lisboa MBMC. Anemia em escolares do ensino público fundamental de Belo Horizonte - MG, 2002 [dissertação de mestrado]. Belo Horizonte: Escola de Enfermagem da Universidade Federal de Minas Gerais; 2002.

23. Boog MCF. Educação nutricional em serviços de saúde. Cad Saúde Pública 1999; 15(S2):139-47.

24. Monteiro CA; Szarfarc SC; Mondini, L. Tendência secular da anemia na infância na cidade de São Paulo (19841996). Rev Saúde Pública 2000; 34(6): 62-72.

25. Oliveira RS, Diniz AS, Benigna MJC, Miranda-Silva SM, Lola MM, Gonçalves MC et al. Magnitude, distribuição espacial e tendência da anemia em pré-escolares da Paraíba. Rev Saúde Pública 2002; 36(1): 26-32.

26. Yip R. Prevention and control of iron deficiency: policy and strategy issues. J Nutr 2002; 132(S4): 8025-55.

27. Nantel G, Tontisirin K. Policy and sustainability issues. $J$ Nutr 2002; 132(S4): 839-44.

28. Garcia-Casal MN, Layrisse M. Iron fortification of flours in Venezuela. Nutr Rev 2002; 60(7 S1): S26-9.

29. Fisberg M. Projeto "Pão é saúde" - Dossiê completo. Belo Horizonte: ABIP (Associação Brasileira da Indústria de Panificação e Confeitaria); 2001.

30. Hurrel R. How to ensure adequate iron absorption from iron-fortified food. Nutr Rev 2002; 60(7 S2): S7-15.

31. Agência Nacional de Vigilância Sanitária. Resolução RDC no 344, de 13 de dezembro de 2002. Brasília: ANVISA; 2002. Disponível em http:/ /www.anvisa.gov.br/ legis/resol/2002/344_02rdc.htm. Acessado em 19 janeiro de 2007.

Recebido em: 13/09/06

Versão final reapresentada em: 06/03/07

Aprovado em: 20/03/07 